\title{
Study of Coronary Flow Reserve with Intravenous Use of Microbubbles (Contrast Echocardiography) and Adenosine. Protocol for Clinical Application in Patients Suspected of Having Coronary Heart Disease
}

\author{
Fernando Morcerf, Alvaro Moraes, Marcia Carrinho, Hans J. F. Dohmann \\ Rio de Janeiro, RJ - Brazil
}

\begin{abstract}
Objective - To test the feasibility, safety and accuracy of the adenosine protocol in the study of myocardial perfusion with microbubbles contrast echocardiography.

Methods - 81 pts (64 male, 60+11 years) were submitted to contrast echocardiography with PESDA (sonicated solution of albumin 20\%-1 ml, dextrose 5\%-12 ml and decafluorobutane gas-8ml) to study the myocardial perfusion at rest and after bolus injection of adenosine (6 to 18mg) and to coronary angiography within 1 month each other. For each patient 3 left ventricle perfusion beds were considered (total of 243 territories). 208 territories were analyzed and 35 territories were excluded. PESDA was continuously infused (1-2ml/min), titrated for best myocardial contrast. Triggered (1:1) second harmonic imaging was used.
\end{abstract}

Results - Coronary angiography showed 70 flow limiting ( $\geq 75 \%$ ) lesions and 138 noflow limiting lesions. At rest an obvious myocardium contrast enhancement was seen in at least 1 segment of a territory in all patients. After adenosine injection an unquestionable further increase in myocardial contrast was observed in 136 territories (99\%) related to no flow limiting lesions, lasting $<10 \mathrm{~s}$, and a myocardial perfusion defect was detected in 68 territories (97\%) related to flow limiting lesions. It was observed only 4 false results. There were no serious complications.

Conclusion - Myocardial perfusion study with PES$D A$ and adenosine protocol is a practical, safe and accurate method to analyze the coronary flow reserve.

Key-words: myocardial perfusion, echocardiographic contrast(microbubbles), coronary disease

Serviço de Ecocardiografia do Hospital Pró-Cardíaco and ECOR - Centro de Diagnóstico Cardiovascular

Mailing address: Fernando Morcerf - Rua Esther Scliar, 51 - 22793-300 - Rio de Janeiro, RJ, Brazil - E-mail: fmorcerf@ cardiol.br

English version by Stela Maris C. e Gandour
Myocardial echocardiographic identification of contrast injected in peripheral veins is a reality ${ }^{1-4}$. Microbubbles obtained with sonication of fluorocarbon gases (PESDA) ${ }^{5-7}$ and other echo contrast agents ${ }^{8-12}$ have already been used. In coronary artery disease with significant obstructions, defects in myocardial perfusion (usually demonstrated in the walls with a reduction in perfusion ${ }^{13}$, mainly after pharmacological stress with dipyridamole ${ }^{14}$ or dobutamine ${ }^{15}$ ) may be useful in identifying myocardial ischemia ${ }^{16}$, myocardial risk area ${ }^{17}$, and in determining infarct size ${ }^{16}$.

The significant reduction in coronary flow reserve resulting from a significant coronary obstruction $(\geq 75 \%)$ is the major marker of myocardial ischemia with physiological significance, because in regions perfused by arteries free from obstruction, this flow may increase up to 3 times its resting value ${ }^{18}$. Examinations using pharmacological stress with dipyridamole or dobutamine may show the decrease in coronary flow reserve, but with mechanisms of action primarily related to other reasons ${ }^{19}$. For both drugs, the appearance of myocardial ischemia is the final effect necessary to show the perfusion defect. These drugs, when used in myocardial perfusion study protocols with intravenous infusion of microbubbles in humans, do not detect the increase in coronary flow in normally perfused walls.

We assessed the hypothesis that intravenous adenosine bolus injection during echocardiography with PESDA microbubbles may not only show the perfusion defect in the hypoperfused coronary bed, but also identify the increase in perfusion in the normal region, due to its promptness in reducing coronary resistance. We also established and tested a protocol for detecting coronary artery disease in humans using echocardiography with PESDA microbubbles at rest and after stress with adenosine.

\section{Methods}

We selected 81 patients (mean age $=60.3 \pm 11$, ranging 
from 30 to 83 years, 64 males and 17 females) out of 532 patients undergoing echocardiography with PESDA microbubbles at the Hospital Pró-Cardíaco and at the ECOR (Centro de Diagnóstico Cardiovascular - Rio de Janeiro). This selection aimed to investigate symptoms suggestive of coronary artery disease in patients who underwent coronary angiography within a month after myocardial contrast echocardiography (tab. I). No patient had aortic stenosis or hypertrophic cardiomyopathy. Fourteen patients had a history of systemic arterial hypertension and 4 had diabetes mellitus. Thirty-one (38.3\%) patients showed some evidence of previous acute myocardial infarction based on their clinical history, on the presence of pathologic $\mathrm{Q}$ waves on electrocardiography at rest, and on the presence of abnormalities in segmentary contraction on left ventriculography and on two-dimensional echocardiography. The left ventricular walls related to the arteries responsible for previous infarcts were excluded from the analysis. In addition to these wal1s, 4 other walls, in 4 different patients, were also excluded because of the low quality of their echocardiographic images. All patients remained clinically stable in the period between the perfusion study and the coronary angiography. Abnormalities in the segmentary contraction at rest were detected in $55(67.9 \%)$ patients, those with previous acute myocardial infarction inclusive (tab. I).

In 9 patients, coronary angiography was considered normal. Of the 72 patients with coronary artery disease, 12

\begin{tabular}{|cc|}
\hline \multicolumn{1}{c|}{$\begin{array}{c}\text { Table I - Clinical, echocardiographic, and coronary angiographic } \\
\text { characteristics of the patients (the numbers in parentheses } \\
\text { represent regions) }\end{array}$} \\
\hline N & 81 patients (243) \\
\hline Age: 30 to 83 years & $60.3 \pm 11$ years \\
Sex: male/female & $64 / 17$ \\
Systemic arterial hypertension & 14 \\
Diabetes mellitus & 4 \\
Abnormal segmentary contraction & 55 \\
Previous AMI & 31 \\
No coronary obstruction & 9 patients (27) \\
No coronary obstruction & 10 patients $(30)$ \\
after revascularization & 1 patient $(3)$ \\
CABG & 4 patients (12) \\
PTCA & 5 patients (15) \\
PTCA + stent & 31 patients $(93)$ \\
Single-vessel disease & $(10)$ \\
Previous AMI & $(1)$ \\
Nonvisualized & $(62)$ \\
Nonlimiting flow & $(20)$ \\
Limiting flow & 19 patients (57) \\
Previous AMI & $(14)$ \\
Nonvisualized & $(1)$ \\
Nonlimiting flow & $(19)$ \\
Limiting flow & $(23)$ \\
Double-vessel diseas & 12 patients (36) \\
Previous AMI & $(7)$ \\
Nonvisualized & $(2)$ \\
Nonlimiting flow & $(0)$ \\
Limiting flow & $(27)$ \\
Triple-vessel disease & \\
AMI- acute myocardial infarction; CABG- coronary artery bypass graft; & \\
PTCA- percutaneous transluminal coronary angioplasty. \\
\hline
\end{tabular}

had disease in 3 vessels, 19 in 2 vessels, 31 in 1 vessel, and 10 patients had no residual coronary lesion after a complete revascularization procedure (implantation of the left internal thoracic artery in 1 patient, percutaneous transluminal angioplasty in 4 patients, and association with stent implantation in 5 patients - tab. I).

In each patient, the left ventricular walls were divided into 3 regions or coronary beds that overlapped. The anterior wall and the anterior part of the interventricular septum were considered part of the region of the anterior descending artery. The anterolateral wall was considered part of the anterior descending artery region or part of the circumflex artery region. The posterolateral wall, the posterior wall, and the posterior part of the interventricular septum were considered part of the circumflex artery region or of the right coronary artery region. Each wall was still divided into 3 segments (basal, middle, and apical) ${ }^{20}$.

In these 81 patients, 70 regions were related to limiting flow coronary lesions (obstruction $\geq 75 \%$ ), 138 regions were related to normal coronary arteries or to nonlimiting flow lesions, 31 regions were related to previous myocardial infarctions, and 4 regions were not visualized. In cases of sequential arterial obstructions, only the most significant one was considered.

Oral consent was obtained for all patients according to the research protocol approved by the National Committee on Ethics and Research of the Health Ministry and by the Committee onEthics and Research of theHospital Pró-Cardíaco.

PESDA was prepared according to the previously reported method ${ }^{21}$ modified to use $20 \%$ human albumin instead of the $5 \%$ solution. Briefly, $1 \mathrm{~mL}$ of $20 \%$ human albumin was mixed with $12 \mathrm{~mL}$ of $5 \%$ glucose solution in a $20-\mathrm{mL}$ syringe connected to a 3 -way tap. Another $20-\mathrm{mL}$ syringe containing $8 \mathrm{~mL}$ of decafluorobutane gas (Flura Corporation, Rock Hill Laboratories, Newport, TN, USA) was then connected to the first syringe. The contents of the 2 syringes were mixed and agitated for at least 1 minute, and then underwent electromechanical sonication for 80 seconds at a frequency of $20 \mathrm{KHz}$, with the sonicator (Heat Systems Inc, Farmingdale, NY, USA) at maximum potency. The resulting solution was maintained in the refrigerator and used within 24 hours.

Patients were advised to restrict their ingestion of caffeinated beverages or theophylline during the 12 hours preceding the examination. Drugs containing dipyridamole were suspended 1 day prior to the examination. No other medication was suspended.

The prepared PESDA solution was diluted in $80 \mathrm{~mL}$ of $5 \%$ glucose solution and continuously infused in a peripheral vein with no infusion pump. Dripping rate depended on the effect obtained with the contrast agent and was performed as follows: we used commercially available equipment (HDI 3000, ATL, Bothell, WA, USA) operating in second harmonic with initial adjustments; intermittent images (trigger mode): 1:1 with the QRS of the electrocardiogram, using T-wave or R-wave peak to obtain the best image; map of the two-dimensional echocardiogram $=8 ;$ dynamic range $=60$ 
$\mathrm{dB}$; output adjusted to produce mechanical index $(\mathrm{mi})=0.7$ or 0.8 ; focus adjusted to the level of the mitral valve in an apical 4-chamber view.

Initial dripping was 30 to 40 drops/minute. After approximately 1 minute, the velocity of infusion was readjusted to provide maximum contrast (close to white in the gray scale) in the left ventricular cavity without production of shadows by the microbubbles. However, if these microbubbles produced a shadow inside the left ventricle, dripping was reduced so that the shadow would remain inside the left atrium, behind the mitral valve, in an apical 4-chamber view.

Obtaining images at rest - After the infusion velocity was established, the controls were adjusted to provide an optimal identification of the contrast agent in the walls (fig. 1) as follows: with a mechanical index of 0.7 or 0.8 (as initially established), the gain was increased until the contrast in the walls was considered satisfactory; time-gain compensation (TGC) was then adjusted to homogenize, from the left ventricular apex to its base, the identification of the contrast in the walls with no difference in the gray scale, mainly in the adjacent regions. Output-power was then diminished step-by-step, reducing the mechanical index, until all segments of the wall became black, allowing, or not, visualization of the maintenance of homogeneity of the contrast agent in the segments of the wall, always comparing segments located within the same distance from the transducer. For example, in the apical 4-chamber view, the basal segment of the lateral wall can only be compared with the basal segment of the interventricular septum; output-power was then increased until the mechanical index of 0.6 or 0.7 was reached. With this adjustment, the contrast in the walls may be perfectly identified, and conditions for its intensification or reduction after adenosine use exist.

After image optimization, all controls of equipment and of PESDA dripping were maintained constant, and the images were recorded on videotape. One ampoule $(6 \mathrm{mg})$ of adenosine (Adenocard $^{\circledR}$, LIBBS Farmacêutica Ltda, São Paulo, Brazil) was then injected in bolus in the same venous route producing a rapid intensification of the contrast agent in at least 1 segment of the left ventricular wall. If no respon-

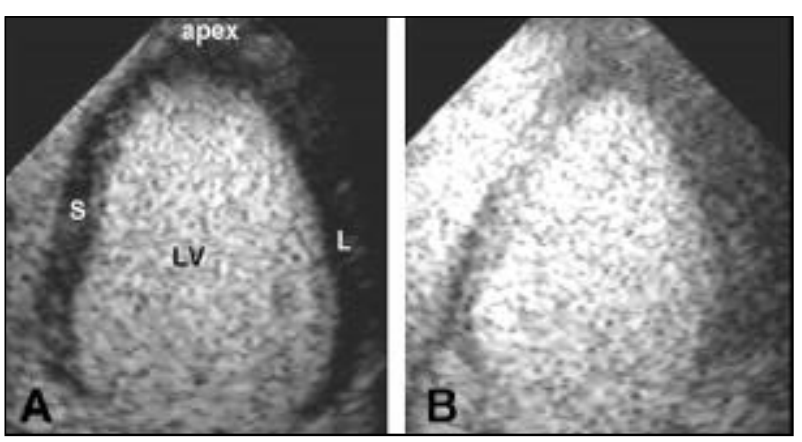

Fig.1 - A) - Prior to final preparation, a great quantity of microbubbles can be seen inside the left ventricle (LV), but not in the walls. Absence of a shadow inside the LV indicates that the dripping velocity of the microbubble solution is satisfactory. B) After final adjustments, the contrast distribution is homogeneous and intense in the walls. L- lateral wall; S- interventricular septum. se was observed, 2 (12mg) or 3 (18mg) ampoules were injected. Adenosine begins to act approximately 20 to 60 seconds after its injection and continues for an additional 20 to 30 seconds. Simultaneously, tachypnea appears and may impede examination. Patients were alerted to the phenomenon and advised to maintain respiratory excursion for at least 5 seconds from the moment they felt the need to inhale deeper.

Echocardiographic imaging was obtained with a specific transducer, which operated at $2 \mathrm{MHz}$ when in the nominal frequency, and at $1.67 \mathrm{MHz}$ for emission and $3.34 \mathrm{MHz}$ for reception, when in second harmonic.

All patients were studied with apical 4-chamber and 2chamber views. Whenever possible, we also used the transverse view of the short left ventricular axis at the level of the papillary muscles, and a modified apical (shortened) view with the ultrasound beam entering in the left ventricular apex and leaving in the inferobasal region.

Image acquisition started right after optimization of the equipment and PESDA dripping controls, and immediately before adenosine injection, continuing until its effect on the myocardium disappeared. Visual gradation of the contrast in the myocardium at rest and of its intensification after adenosine bolus injection was performed by 2 independent reviewers, based on videotape images in loop format using the computerized system ImageVue DCR (Nova MicroSonics, Allendale, NJ, USA). Each reviewer assessed the distribution and homogeneity of the contrast, at rest, in each left ventricular wall and in all echocardiographic views used. After adenosine injection, we assessed intensification, reduction, or maintenance of the intensity of the contrast agent in the walls, which could be observed during the maximum effect of the drug.

The normal myocardial perfusion image at rest was present when the contrast, in a certain echocardiographic view, had a homogeneous distribution in all segments of the left ventricular walls; comparison was always performed between segments situated at the same depth in a given echocardiographic view (fig. 1). Persistence of this homogeneity with a decrease in the mechanical index was normal until the contrast agent could no longer be observed in the myocardium (fig. 2) in all images obtained. After adenosine bolus injection, the normal perfusion image had a marked intensification of the contrast agent in all left ventricular wall segments (fig. 3).

The myocardial perfusion image at rest was considered abnormal when the contrast agent was heterogeneously distributed in the left ventricular wall segments (fig. 4), or when this heterogeneity could be induced or better demonstrated with a decrease in the mechanical index, a condition in which the abnormal segment would appear darker than its contralateral segment (fig. 5). After adenosine injection, the abnormal perfusion image showed lack of intensification of the contrast agent in defective segments at rest (fig. 6) or, as more frequently observed, a reduction in the contrast agent in normally or poorly contrasted segments at rest (fig. 7).

Interobserver and intraobserver variability in the analysis of the perfusion images reported was determined by 


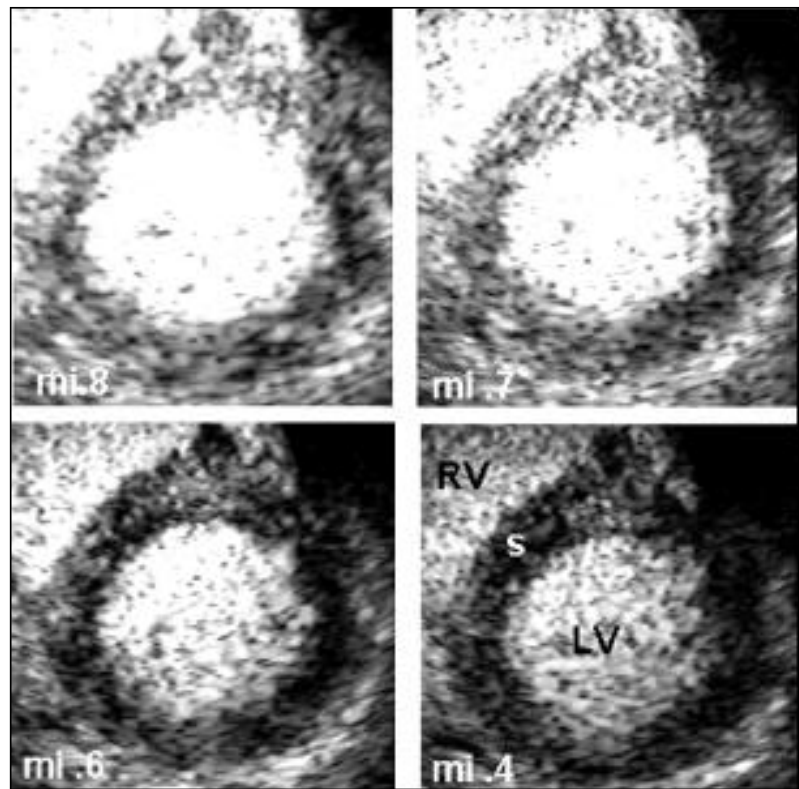

Fig. 2 - Persistence of homogeneity in all images obtained during the gradual reduction in the mechanical index (mi) until no more contrast agent can be seen in the walls. LV- left ventricle; RV- right ventricle; $\mathrm{S}$ - interventricular septum.

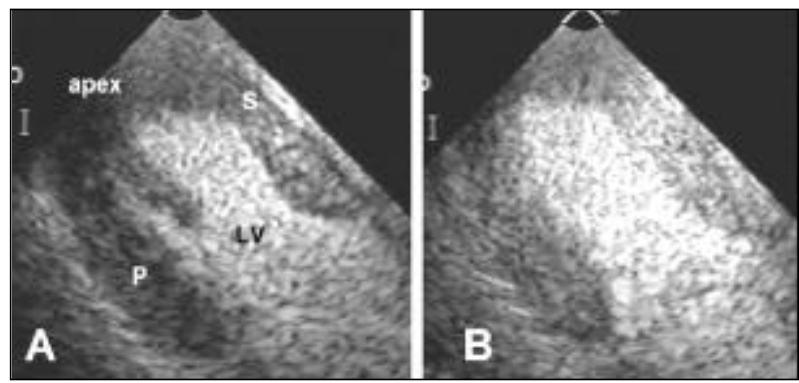

Fig. 3 - Normal pattern of myocardial perfusion: before (A) and after (B) adenosine bolus injection. The normal pattern of perfusion is the marked intensification of the contrast agent observed at rest in all segments of the LV walls. P- posterior wall; Sinterventricular septum; $\mathrm{LV}$ - left ventricle.

comparison of the percentage of concordance between the 2 independent observers in all patients.

Statistical analysis - To determine inter-and intraobserver variability, we used the kappa $(\mathrm{k})$ statistic with values greater than 0.4, 0.6, and 0.8 indicating low, good, and excellent concordances, respectively ${ }^{22}$. The chi-square test was used to determine the significance or nonsignificance of the distribution of concordance between coronary obstruction and the perfusion defect, and also between the absence of coronary obstruction and normal perfusion. Sensitivity, specificity, global accuracy, and positive and negative values of the examination were also calculated ${ }^{23}$. The Student $t$ test for pared samples was used to compare the means of heart rate before and after adenosine bolus injection. For all statistical tests, a value lower than 0.05 was considered significant.

\section{Results}

Intravenous infusion of PESDA produced myocardial

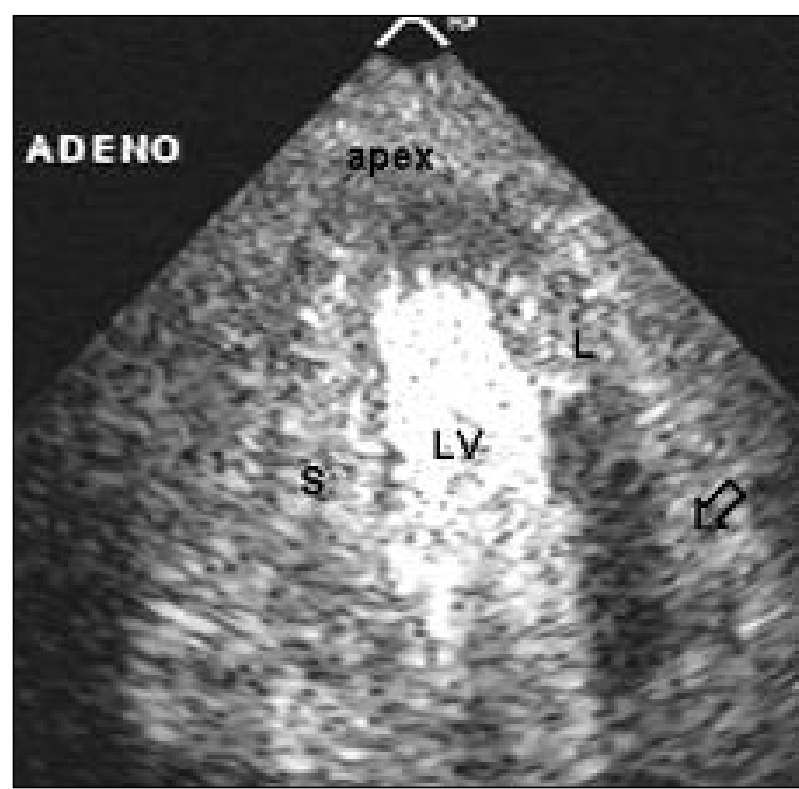

Fig. 4 - Evident perfusion abnormality at rest, prior to adenosine administration, in the basal part of the lateral wall (arrow). L- lateral wall; S- interventricular septum; LV- left ventricle.

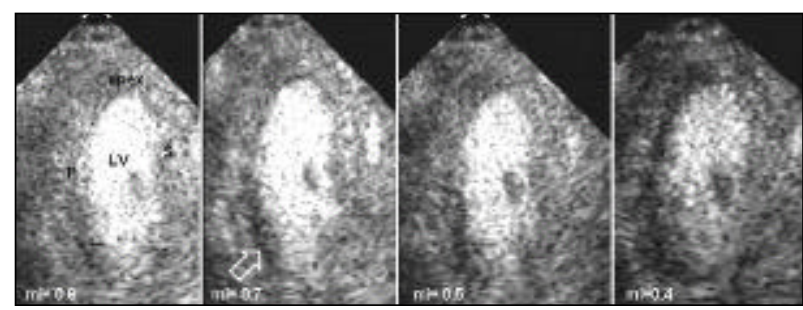

Fig. 5 -Heterogeneous pattern of myocardial perfusion at rest induced by gradual reduction in themechanical index (mi). Themechanical index reduction from 0.8 to 0.7 documents theperfusion abnormality in the basal part of the posterior wall, and the progression in mechanical index reduction down to 0.4 documents the perfusion abnormality in all the wall extension. $\mathrm{P}$ posterior wall; $\mathrm{S}$ - interventricular septum; LV-left ventricle.
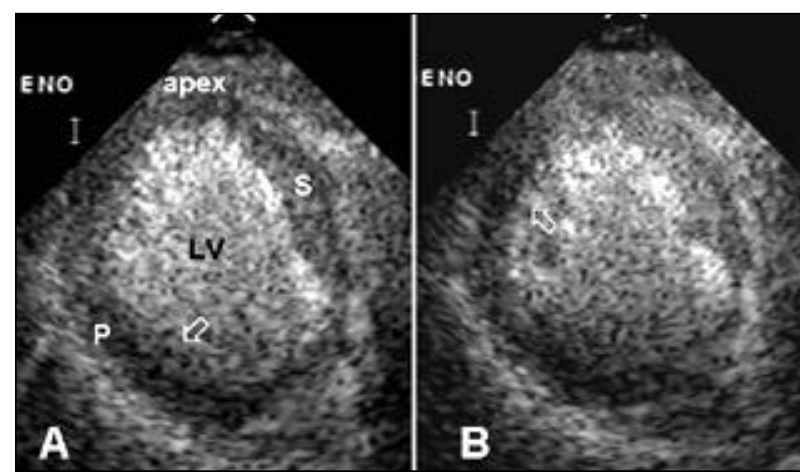

Fig. 6 - At rest (A), perfusion abnormality is seen in the basal part of the posterior wall (arrow). After adenosine administration (B), the abnormal region becomes darker and can be seen spreading to the apical part of the posterior wall (arrow). P - posterior wall; S - interventricular septum; LV - left ventricle.

contrast, which was intensified by the adenosine bolus injection in at least 1 segment of the left ventricular walls, in all patients when visually estimated by 2 reviewers. 


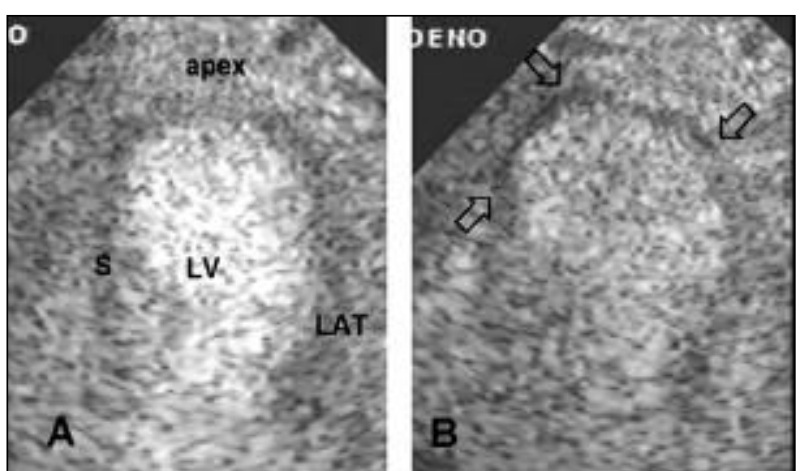

Fig. 7 - Homogeneous perfusion (normal) at rest (A). After adenosine administration (B), the perfusion abnormality (dark area) can be seen in the apex and apical region of the interventricular septum. S - interventricular septum; Lat - lateral wall; LV - left ventricle.

To obtain contrast intensification in the left ventricular walls, $62(76.5 \%)$ patients required 1 single ampoule $(6 \mathrm{mg})$ of adenosine, $14(17.3 \%)$ patients required $12 \mathrm{mg}$, and $5(6.2 \%)$ patients required $18 \mathrm{mg}$ to obtain the same result.

In these 81 patients, a total of 208 regions not associated with infarcts related to obstructed coronary arteries were analyzed (tab. II and fig. 8). We observed that 138 coronary arteries (or regions) were normal or had an obstruction $<75 \%$, and that 70 coronary arteries (or regions) had a severe obstruction (lesion $\geq 75 \%$ ). Of these 70 obstructions, impairment of the area of the anterior descending coronary artery was observed in 18 patients, of the area of the circumflex artery or of the obtuse marginal artery in 33 patients, and of the area of the right coronary artery in 19 patients.

The visual pattern of normal perfusion at rest and after adenosine bolus injection was observed in 136 of the 138 regions $(98.6 \%)$ perfused by a normal coronary artery or an artery with obstruction $<75 \%$. In the 2 other regions, the perfusion pattern was considered abnormal (false-positive).

In the 70 regions perfused by arteries with total obstruction or obstruction $\geq 75 \%$, as compared with the normally perfused segments, a reduction in the intensification of the contrast agent at rest was observed (consensus between the 2 reviewers) in at least 1 segment in 52 (74.3\%) regions. In regard to these regions, adenosine bolus injection increased the abnormal area in $20(38.5 \%)$ regions, but did not change the abnormal area in the remaining $32(61.5 \%)$ regions. In $16(22.9 \%)$ normally perfused regions at rest, an

\begin{tabular}{|c|c|c|c|c|c|}
\hline \multicolumn{6}{|c|}{ Table II - Distribution of the regions analyzed and excluded } \\
\hline \multicolumn{3}{|c|}{ Analyzed $=208$} & \multicolumn{3}{|c|}{ Excluded $=35$} \\
\hline $\mathrm{AD}=67$ & $\mathrm{Cx}=78$ & $\mathrm{RC}=59$ & $\mathrm{AD}=12$ & $\mathrm{Cx}=2$ & $\mathrm{RC}=21$ \\
\hline NL AN & NL AN & NL AN & nv fib & nv fib & nv fib \\
\hline $50 \quad 17$ & $45 \quad 33$ & $41 \quad 18$ & 48 & 02 & $0 \quad 21$ \\
\hline 1FN@1FP\& & $-1 F P \&$ & IFN@- & $-\quad-$ & $-\quad-$ & $-\quad-$ \\
\hline \multicolumn{6}{|c|}{$\begin{array}{l}\text { AD- anterior descending artery; } \mathrm{Cx} \text { - circumflex artery; RC- right coronary } \\
\text { artery; NL- normal perfusion; AN- abnormal perfusion; nv- nonvisualized; } \\
\text { fib- fibrosis; } F N \text { - false-negative; } F P \text { - false-positive; @ - related to the same } \\
\text { patient; \& - } 2 \text { different patients. }\end{array}$} \\
\hline
\end{tabular}

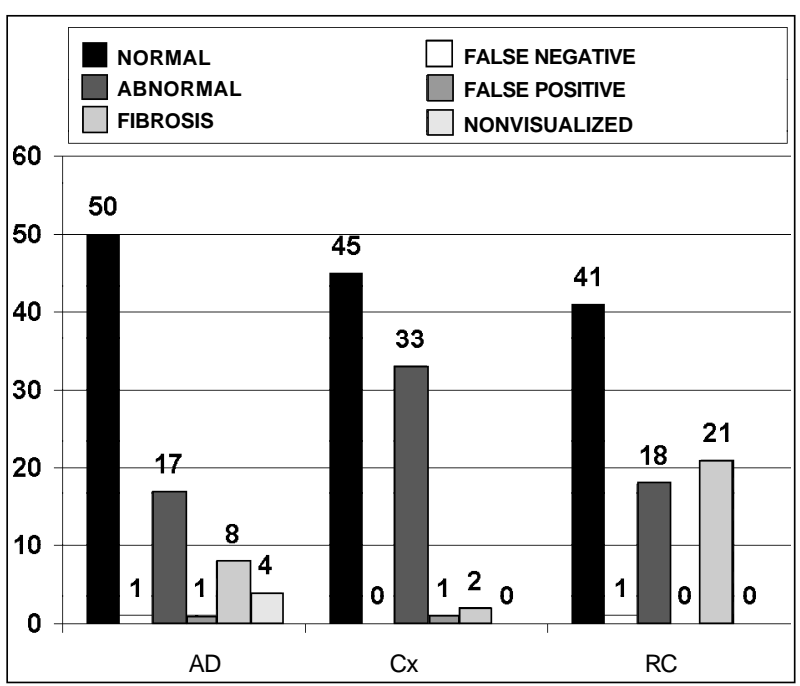

Fig. 8 - Distribution of the myocardial perfusion patterns in the region-by-region analysis. AD - anterior descending artery; $\mathrm{Cx}$ - circumflex artery; $\mathrm{RC}$ - right coronary artery.

abnormality was only demonstrated after adenosine bolus injection. Only 2 regions showed the normal perfusion pattern at rest and after adenosine injection (false-negative).

Sensitivity was $97.1 \%$ (68 of the 70 regions related to arteries with limiting flow obstructions); specificity was $98.6 \%$ (136 of the 138 regions related to normal arteries or arteries with nonlimiting flow obstruction); and global accuracy was $98.1 \%$ (204 of the 208 regions analyzed). Positive and negative predictive values were $97.1 \%$ and $98.6 \%$, respectively. The odds ratio for an abnormal test was 0.694 , and 0.34 for a normal test $(\mathrm{p}<0.001)$.

Concordance between absence or presence of coronary obstruction and normal or abnormal perfusion using the chi-square test was statistically significant $\left(\chi^{2}=186.21\right.$, $\mathrm{p}<0.001$ ), with only 4 discordant cases ( 2 in normal regions and 2 in abnormal regions). Intra- and interobserver concordances were excellent, with $\mathrm{k}=0.94$ and $\mathrm{k}=0.91$, respectively.

Adenosine, at the dosages used, did not cause abnormalities in segmentary contraction in any patient. On the other hand, it induced a mild, but significant, increase in heart rate (from $71 \pm 8$ to $84 \pm 14 \mathrm{bpm}, \mathrm{p}<0.001$ ). Two patients who received $12 \mathrm{mg}$ and 1 who received $18 \mathrm{mg}$ of adenosine had asymptomatic and transient (8 to 10 seconds) third-degree atrioventricular block. The sensation of facial heat, chest discomfort, and cephalea were reported in 10 (12\%), 3 $(3.7 \%)$, and $4(4.9 \%)$ patients, respectively. All patients developed tachypnea, which lasted less than 30 seconds, did not require treatment, and did not impede new adenosine injections when necessary.

\section{Discussion}

This study shows that myocardial contrast echocardiography with continuous PESDA infusion and adenosine bolus injection may assess coronary flow reserve, and, therefore, may be used to identify coronary artery disease, be- 
cause the abnormal perfusion sites correlate with the regions related to obstructed arteries.

The severity of coronary obstruction assessed on coronary angiography is usually expressed as a percentage of stenosis in relation to the diameter of the vessel. This method has limitations, because it does not take into consideration other characteristics of the lesion, such as length, shape, and eccentricity, which are known to affect coronary flow resistance. The weak correlation existing between the coronary angiographic assessment of the degree of obstruction and the postmortem examination findings is also known ${ }^{24-26}$. A great intra- and interobserver variation exists ${ }^{27-29}$, as well as a limited capacity of the observers to predict the hemodynamic significance of a certain coronary stenosis ${ }^{30,31}$. Quantitative coronary angiography improved the evaluation of the degree of coronary stenosis ${ }^{32}$, but limitations still persist $^{33,34}$. In addition, the demonstration of the stenosis and its degree does not describe the level of the perfusion defect, or the myocardial area involved.

Analysis of coronary flow reserve should be used to assess the hemodynamic consequences of coronary artery stenosis ${ }^{35-37}$. The measurement of coronary flow reserve during quantitative coronary angiography has been proposed to assess all anatomical factors that influence the severity of arterial stenosis ${ }^{38,39}$. A good correlation between coronary flow reserve analyzed on intracoronary Doppler and the arterial stenosis detected on quantitative coronary angiography has also been reported ${ }^{40}$. Myocardial contrast echocardiography has also been proposed to assess the physiological significance of coronary lesions during pharmacological vasodilation induction ${ }^{41-45}$.

Adenosine is a potent coronary vasodilator. Peripheral venous adenosine injection increases coronary flow 3 to 4 times due to a drop in coronary resistance ${ }^{46}$, which results in an increase in myocardial vascularization and in myocardial blood flow. In coronary artery disease with single-vessel obstruction, adenosine injection increases blood flow in normally perfused regions, where resistance will decrease. In the areas perfused by an artery with limiting flow obstruction, the resistance vessels are maximally dilated and no changes will occur, or a reduction in flow may happen due to the coronary steal phenomenon ${ }^{47-49}$.

Variation in the intensity of the contrast reflects changes in myocardial blood flow ${ }^{50}$. Therefore, an increase in the intensity of the contrast is expected to occur in the absence of coronary artery stenosis. In our patients, this fact was observed in 136 of the 138 regions perfused by normal coronary arteries or by coronary arteries with nonsignificant obstructions. However, in cases of significant coronary stenosis, coronary flow reserve is reduced and the response to adenosine injection (vasodilation and capillary recruitment) is not observed, and, therefore, the normal increase in myocardial blood volume does not occur ${ }^{50}$. Therefore, the same increase in contrast intensity observed in normal regions did not occur in 68 of the 70 regions perfused by coronary arteries with significant obstructions. In our study, the use of these perfusion patterns had a global accuracy of $98.1 \%$, with high positive and negative predictive values (97.1\% and $98.6 \%$, respectively).

Despite the alterations in the distribution of coronary flow, adenosine injection does not necessarily induce myocardial ischemia, even when it causes abnormalities in myocardial blood volume and makes the intensification of myocardial contrast in the abnormal segments heterogeneous ${ }^{51}$.

Adenosine bolus injection caused a marked difference (easily identified from the point of view of visual assessment) between the lowest myocardial contrast observed in the region perfused by a critically stenotic vessel and the intensification of the contrast seen in the region supplied by a normal vessel or a noncritically stenotic vessel. These data are in accordance with the fact that coronary flow reserve is reduced in the coronary bed related to a critical obstruction when compared with that related to the normally perfused coronary bed.

If in a certain territory the intensity of contrast demonstrated at rest does not increase after adenosine or diminishes, as frequently noted, one may infer that that region is supplied by a critically stenotic artery. This observation is supported by a study by Lang et al ${ }^{52}$, who showed that the increase in the intensity of contrast is greater in the regions related to obstructed arteries that undergo angioplasty than in regions related to normal arteries due to subsequent hyperemia.

Techniques with pharmacological stress are widely used to diagnose coronary artery disease ${ }^{53-58}$. Dipyridamole is one of the most used drugs to cause myocardial ischemia with segmentary contraction abnormalities on two-dimensional echocardiography ${ }^{58}$, with sensitivity similar to that of thallium-201 myocardial perfusion scintigraphy. With this drug, which has a relatively long persistence, episodes of severe ischemia, myocardial infarction, and even death, though rare, have been reported ${ }^{58-60}$.

Coronary vasodilation produced ley dipyridamole is due to its blocking effect on cellular uptake of adenosine, which results in an increase in endogenous adenosine, therefore potentiating its vasodilating effect ${ }^{61,62}$. Intravenous adenosine, which has a very short half-life ( $<20$ seconds), has been used as an alternative to dipyridamole as a potent coronary vasodilator. The protocols for pharmacological stress tests with adenosine recommend the continuous infusion of the drug at the dosage of $0.14 \mathrm{mg} / \mathrm{kg} / \mathrm{min}^{63-65}$. The side effects observed with adenosine are of short duration, but much more frequent and less well tolerated than those occurring with dipyridamole ${ }^{64}$. These side effects impede obtainment of a maximum test in approximately $25 \%$ of patients ${ }^{65}$. This high proportion of submaximum studies diminishes the sensitivity of adenosine echocardiography, when the objective is to cause ischemia with the appearance of segmentary contraction alteration. However, as in this protocol, adenosine is administered in intravenous bolus, and, consequently, with a very short persistence, stress does not cause imbalance in myocardial oxygen delivery and consumption for a time long enough to induce segmentary 
contraction abnormalities. A regional mismatch occurs in myocardial perfusion, which becomes heterogeneous when a region depends on a coronary artery with critical stenosis.

Several clinical trials have determined the sensitivity and specificity of myocardial perfusion during adenosine infusion using thallium-201 scintigraphy ${ }^{66-69}$ or the alteration in segmentary contraction of the left ventricular walls with echocardiography ${ }^{63,70}$. Three studies have compared sensitivity and specificity in myocardial perfusion imaging using adenosine infusion and dipyridamole ${ }^{38,70,71}$. In all these studies, adenosine was administered as an intravenous infusion, and no increase in the intensity of myocardial contrast was observed in the regions related to normal arteries. As far as we know, this is the first report on the effect of adenosine, when administered in intravenous bolus, on coronary flow reserves during myocardial contrast echocardiography.

Adenosine injection in bolus caused total and asymptomatic transient (less than 10 seconds of duration) atrioventricular block in 3 of the 81 patients of this study. However, atrioventricular blocks with no sequelae ${ }^{72,73}$ and a high incidence of other side effects ${ }^{74,75}$ have also been reported during adenosine infusion. Chest pain, headache, facial rubor, and dyspnea may more often occur with adenosine than with dipyridamole ${ }^{76}$. After adenosine bolus injection, these effects were very discrete and transitory, completely disappearing in 20 to 30 seconds, unlike those resulting from dipyridamole administration, which last longer and may require the intravenous antidote (aminophylline).

The results of this study may have been influenced by some methodological limitations.

We compared the results of myocardial perfusion obtained through microbubble echocardiography with those obtained through coronary angiography in regard to the presence or absence of intensification of the myocardial contrast and critical $(\geq 75 \%)$ or noncritical $(<75 \%)$ coronary artery obstruction, not considering the continuous spectrum of the severity of obstruction.

The reduction in the capacity of arterial vasodilation and in coronary flow reserve has been shown to increase as the severity of coronary stenosis increases ${ }^{77,78}$. A previous study ${ }^{79}$ inferred that the relative, more than the absolute, estimation of myocardial perfusion reserve would be a more sensitive physiological indicator than the severity of arterial stenosis on coronary angiography.

Anatomical factors of coronary lesions, such as the shape and eccentricity of the obstructions, and the presence of serial stenoses, which may affect coronary flow resistance, were not considered.

Another limitation of this study was the use of qualitative and not quantitative estimations of coronary flow resistance. However, quantitative assessments also have significant restrictions ${ }^{80}$.

Finally, the high global accuracy with very high positive and negative values is, at least in part, due to the high incidence of coronary artery disease in our patients. The values calculated for the odds ratio (normal test $=0.34$ and $a b$ normal test $=0.694$ ) confirm this observation.

In summary, in patients with coronary artery disease, qualitative noninvasive assessment of coronary flow reserve using myocardial perfusion echocardiography with microbubbles, before and after adenosine bolus injection, is a safe method that is closely related to the presence of critical coronary obstructions detected on coronary angiography. This information may provide a better definition of the functional relevance of an already known coronary lesion. In addition, this method may be clinically useful for detecting silent ischemia, for monitoring progression or regression of coronary obstructions, and for assessing revascularization procedures. The short persistence and clearance of adenosine may reduce the time necessary for examination, and also reduce the duration of any side effects that may appear.

\section{References}

1. Villanueva F, Glasheen WP, Sklenar J, Jayaweera AR, Kaul S. Successful and reproducible myocardial opacification during two-dimensional echocardiography from right heart injection of contrast. Circulation 1992; 85: 1557-64.

2. Cotter B, Kriett J, Perricone T, et al. Detection of coronary artery occlusion by decreased myocardial opacification following intravenous injection of QW3600 (EchoGen). Circulation 1994; 90(Pt 2): I-67.

3. Camarano GP, Ismail S, Goodman NC, Kaul S. Assessment of risk area during coronary occlusion and infarct size after reperfusion can be determined with myocardial contrast echocardiography using intravenous injections of FS-069, a new contrast agent. Circulation 1994; 90(Pt 2): I-68.

4. Grauer S, Pantely GA, Xu J, et al. Aerosomes MRX 115: echocardiographic and hemodynamic characteristics of new echo contrast agent that produces myocardial opacification after intravenous injections in pigs. Circulation 1994; 90(Pt 2): I-556.

5. Xie F, Porter TR. Acute myocardial ischemia and reperfusion can be visually identified non-invasively with intravenous perfluoropropane-enhanced sonicated albumin ultrasound contrast. Circulation 1994; 90: I-555.

6. Morcerf F, Moraes A, Nogueira AC, et al. Myocardial contrast echocardiography with intravenous microbubbles and adenosine bolus injection: a new method to identify myocardial perfusion defects in humans. J Am Coll Cardiol 1998; 31(suppl C): 273C.

7. Morcerf F, Moraes A, Nogueira AC, et al. Myocardial contrast echocardiography: normal perfusion pattern in humans with triggered second harmonic imaging mode following intravenous infusion of microbubbles. J Am Coll Cardiol 1998; 31(suppl C): 273C.

8. Meza M, Greener Y, Hunt R, et al. Myocardial contrast echocardiography: reliable, safe, and efficacious myocardial perfusion assessment after intravenous injections of a new echocardiographic contrast agent. Am Heart J 1996; 132: 871-81.

9. Meza M, Greener Y, Aristizabal D, et al. Myocardial contrast echocardiography safety of FS069, a new transpulmonary echocardiographic contrast agent. J Am Soc Echocardiogr 1994; 7: S36.

10. Dittrich H, Bales G, Kuvelas T, Hunt R, McFerran B, Greener Y. Myocardial contrast echocardiography in experimental coronary artery occlusion with a new intravenously administered contrast agent. J Am Soc Echocardiogr 1995; 8: 465-74.

11. Grayburn PA, Erikson JM, Velasco CE. Assessment of myocardial risk area and infarct size by peripheral intravenous injection of a new phase-shift echo contrast agent. Circulation 1994; 90: I-555. 
12. Grauer S, Panteli GA, Xu J, et al. Aerosomes MRX 115: echocardiographic and hemodynamic characteristics of a new echo contrast agent that produces myocardial opacification after intravenous injection in pigs. Circulation 1994; 90: I- 556.

13. Moraes A, Morcerf F, Nogueira AC, et al. Myocardial contrast echocardiography for the detection of coronary artery disease. J Am Coll Cardiol 1998; 31(suppl C): 272C.

14. MorcerfF, Castier M, SalekF, Dohmann HFJ, Moraes A. Myocardial contrast imaging with decafluorobutane microbubbles: an attempt to develop a suitable protocol for clinical application. Circulation 1997; 96(suppl I): I- 457.

15. Porter TR, Xie F, Kricsfeld A, Deligonul U, Kilzer K, Kricsfeld D. Myocardial perfusion abnormalities during low-dose dobutamine after coronary reperfusion can be demonstrated with intravenous perfluorocarbon-exposed sonicated dextrose albumin ultrasound contrast. Am Heart J 1996; 131: 1079-87.

16. Villanueva F, Glasheen WP, Sklenar J, Kaul S. Assessment of risk area coronary occlusion and infarct size after reperfusion with myocardial contrast echocardiography using left and right atrial injections of contrast. Circulation 1993; 88: 596-604.

17. Grayburn PA, Erikson JM, Velasco CE. Assessment of myocardial risk area and infarct size by peripheral intravenous injection of a new phase shift echo contrast agent. Circulation 1994; 90: I-555.

18. Keller MW, Glasheen W, Smucker ML, Burwell LR, Watson DD, Kaul S. Myocardial contrast echocardiography in humans I: assessment of coronary blood flow reserve. J Am Coll Cardiol 1988; 12: 925-34.

19. Picano E. Stress Echocardiography. $2^{\text {nd }}$ ed. Berlin, Heidelberg, New York: Springler-Verlag, 1994.

20. Morcerf F, Cantisano A, Salek F, et al. Myocardial contrast echocardiography (MCE): safety and normal perfusion pattern in humans. J Am Coll Cardiol 1993; 21(suppl A): 347A.

21. Porter TR, Xie F. Transient myocardial contrast following initial exposure to diagnostic ultrasound pressures with minute doses of intravenously injected microbubbles: demonstration and potential mechanisms. Circulation 1995; 92: 2391-5.

22. Kramer MS, Feinstein AR. Clinical biostatistics: the biostatistics of concordance. Clin Pharmacol Ther 1981; 29: 111-23.

23. Chaitman BR. Exercise Stress Testing. In: Braunwald E, ed. Heart Disease. A Textbook of Cardiovascular Medicine. $5^{\text {th }}$ ed. Philadelphia: WB Saunders Co., 1997: 161.

24. VlodaverZ, French R, Van Tassel RA, Edwards JE. Correlation of the antemortem angiogram and the postmortem specimen. Circulation 1973; 47: 162-9.

25. Arnett EM, Isner JM, Redwood DR, et al. Coronary artery narrowing in coronary artery disease: comparison of cineangiography and necropsy findings. Ann Intern Med 1979; 91: 350-6.

26. Schwartz JN, Kong Y, Hackel DB, Hackel DB. Comparison of angiocardiographic and postmortem findings in patients with coronary artery disease. Am J Cardiol 1975; 36: 474-8.

27. DeRouen TA, Murray JA, Owen W. Variability in the analysis of coronary arteriograms. Circulation 1977; 55: 324-8.

28. Detre KM, Wright E, Murphy ML, Takaro T. Observer agreement in evaluating coronary angiograms. Circulation 1975; 52: 979-86.

29. Zir LM, Miller SW, Dinsmore RE, Gilbert JP, Harthorne JW. Interobserver variability in coronary angiography. Circulation 1976; 53: 627-32.

30. Klocke FJ. Measurements of coronary blood flow and degree of stenosis: current clinical implications and continuing uncertainties. J Am Coll Cardiol 1983; 1: 31-41.

31. White CW, Wright CB, Doty DB, et al. Does visual interpretation of the coronary arteriogram predict the physiologic importance of a coronary stenosis? N Engl J Med 1984; 310: 819-24.

32. Wilson RF, Marcus ML, White CW. Prediction of the physiologic significance of coronary arterial lesions by quantitative lesion geometry in patients with limited coronary artery disease. Circulation $1987 ; 75: 723-32$.

33. Gould KL, Kelley KO. Physiologic significance of coronary flow velocity and changing stenosis geometry during vasodilation in awake dogs. Circ Res 1982; 50: 695-704.

34. Gould KL, Kelley KO, Bolson EL. Experimental validation of quantitative coronary arteriography for determining pressure-flow characteristics of coronary stenosis. Circulation 1982; 66: 930-7.

35. Gould KL, Lipscomb K, Hamilton GW. Physiologic basis for assessing critical coronary stenosis: instantaneous flow response and regional distribution during coronary hyperemia as measures of coronary blood flow reserve. Am J Cardiol 1974; 33: 87-94.

36. Wilson RF, White CW. Intracoronary papaverine: an ideal coronary vasodilator for studies of the coronary circulation in conscious humans. Circulation 1986; 73: 444-51.

37. Nissen SE, Elion JL, Booth DC, Evans J, DeMaria AN. Value and limitations of computer analysis in the assessment of coronary flow reserve. Circulation 1986; 73: 562-71.

38. Kirkeeide RL, Gould KL, Parsel L. Assessment of coronary stenosis by myocar- dial perfusion imaging during pharmacological vasodilatation, VII: validation of coronary flow reserve as a single integrated functional measure of stenosis severity reflecting all its geometric dimensions. J Am Coll Cardiol 1986; 7: 103-13.

39. Gould KL, Goldstein RA, Mullani NA, et al. Noninvasive assessment of coronary stenosis by myocardial perfusion imaging during pharmacological coronary vasodilatation, VIII: clinical feasibility of positron cardiac imaging without a cyclotron using a generator-produced rubidium-82. J Am Coll Cardiol 1986; 7 : 775-89.

40. Wilson RF, Marcus ML, White CW. Prediction of the physiologic significance of coronary arterial lesions by quantitative lesion geometry in patients with limited coronary artery disease. Circulation 1987; 75: 723-32.

41. Gould KL. Noninvasive assessment of coronary stenosis by myocardial perfusion imaging during pharmacological coronary vasodilatation, I: physiologic basis and experimental validation. Am J Cardiol 1978; 41: 267-8.

42. Albro PC, Gould KL, Westcott RJ, Hamilton GW, Ritchie JL, Williams DL. Noninvasive assessment of coronary stenosis by myocardial perfusion imaging during pharmacologic coronary vasodilatation, III: clinical trial. Am J Cardiol 1978; 42: 751-60.

43. Gould KL. Assessment of coronary stenosis with myocardial perfusion imaging during pharmacologic coronary vasodilation, IV: limits of detection of stenosis with idealized experimental cross-sectional myocardial imaging. Am J Cardiol 1978; 42: 761-8.

44. Gould KL, Schelbert HR, Phelps ME, Hoffman EJ. Noninvasive assessment of coronary stenosis with myocardial perfusion imaging during pharmacologic coronary vasodilation, $\mathrm{V}$ : detection of $47 \%$ diameter coronary stenosis with intravenous N-13 ammonia and positron emission tomography in intact dogs. Am J Cardiol 1979; 43: 200-8.

45. Schelbert HR, Wisenberg G, Phelps ME, et al. Noninvasive assessment of coronary stenosis with myocardial perfusion imaging during pharmacologic coronary vasodilation, VI: detection of coronary artery disease in human beings with intravenous N-13 ammonia and positron computed tomography. Am J Cardiol 1982; 49: 1197-207.

46. Verani MS. Pharmacologic stress myocardial perfusion imaging. Curr Probl Cardiol 1993; 18: 481-528.

47. Patterson RE, Kirk ES. Coronary steal mechanisms in dogs with one-vessel occlusion and other arteries normal. Circulation 1983; 67: 1009-15.

48. Ofili EO, Dressler FA, St. Vrain JA, et al. Pharmacologic stress-induced regional myocardial blood flow heterogeneity and left ventricular wall thickening abnormality: comparison of intravenous adenosine with dipyridamole in a model of critical coronary stenosis. Am Heart J 1997; 133: 78-86.

49. Becker LC. Conditions for vasodilator-induced coronary steal in experimental myocardial ischemia. Circulation 1979; 57: 1103-10.

50. Kaul S, Jayaweera AR. Coronary and myocardial blood volumes: noninvasive tools to assess the coronary microcirculation? Circulation 1997; 96: 719-24.

51. Rovai D, DeMaria AN, L'Abbate A. Myocardial contrast echo effect: the dilemma of coronary blood flow and volume. J Am Coll Cardiol 1995; 26: 12-7.

52. Lang RM, Feinstein SB, Feldman T, Newmann A, Chua KG, Borow KM. Contrast echocardiography for evaluation of myocardial perfusion: effects of coronary angioplasty. J Am Coll Cardiol 1986; 8: 232-5.

53. Leppo JA, Boucher CA, Okada RD, Newell JB, Strauss WH, Pohost GM. Serial thallium 201 myocardial imaging after dipyridamole infusion: diagnostic utility in detecting coronary stenosis and relationship to regional wall motion. Circulation 1982; 66: 649-56.

54. Stratman HG, Kennedy HL. Evaluation of coronary artery disease in the patient unable to exercise: alternatives to exercise testing. Am Heart J 1989; 117: 1344-65.

55. Boucher CA, Brewster DC, Darling JA, Okada RD, Shauss HW, Pohost GM. Determination of cardiac risk of dipyridamole thallium imaging before peripheral vascular surgery. N Engl J Med 1985; 312: 389-94.

56. Lam J, Chaitman B, Glaenzer M, et al. Safety and diagnostic accuracy of dipyridamole-thallium imaging in the elderly. J Am Coll Cardiol 1988; 11: 585-9.

57. Kong B, Shaw L, Miller D, Chaitman B. Comparison of accuracy for detecting coronary artery disease and side-effect profile of dipyridamole thallium-201 myocardial perfusion imaging in women versus men. Am J Cardiol 1992; 70: 168-73.

58. Picano E, Marini C, Pirelli S, et al. Safety of intravenous high-dose dipyridamole echocardiography. Am J Cardiol 1992; 70: 252-8.

59. Lewen MK, Labovitz AJ, Kern MJ, Chaitman BR. Prolonged myocardial ischemia after intravenous dipyridamole thallium imaging. Chest 1987; 92: 1102-4.

60. Ranhosky A, Kempthorne-Rawon J, IDTIS Group. The safety of intravenous dipyridamole thallium myocardial perfusion imaging. Circulation 1990; 81: 1205-9.

61. Freilich A, Tepper D. Adenosine and its cardiovascular effects. Am Heart J 1992; 123: 1324-8.

62. Belerdinelli L, Linden J, Berne RM. The cardiac effects of adenosine. Prog Cardiovasc Dis 1989; 32: 73-97.

63. Zoghbi WA, Cheirif J, Kleiman NS, Verani MS, Trakhtenbroit A. Diagnosis of ischemic heart disease with adenosine echocardiography. J Am Coll Cardiol 1991; 18: 1271-9. 
64. Martin T, Seaworth J, Johns J, Pupa L, Condos W. Comparison of adenosine, dipyridamole and dobutamine stress echocardiography for the detection of coronary artery disease. J Am Coll Cardiol 1991; 17: 277A.

65. Marwick T, Willemart B, D'Hondt AM, et al. Selection of the optimal non-exercise stress for the evaluation of ischemic regional myocardial dysfunction and malperfusion: comparison of dobutamine and adenosine using echocardiography and Tc-99m MIBI single photon emission computed tomography. Circulation 1993; 87: $345-54$

66. Mahmarian JJ, Hixson JB, Boyce BS, Staudacher RA. Diagnosis of coronary artery disease by controlled coronary vasodilation with adenosine and thallium201 scintigraphy in patients unable to exercise. Circulation 1990; 82: 80-7.

67. Gupta NC, Esterbrooks DJ, Hilleman DE, Mohiuddin SM. Comparison of adenosine and exercise thallium-201 single photon emission computer tomography (SPECT) myocardial perfusion imaging. J Am Coll Cardiol 1992; 19: 248-57.

68. Verani MS, Mahmarian JJ, Hixson JB, Boyce TM, Staudacher RA. Diagnosis of coronary artery disease by controlled coronary vasodilation with adenosine and thallium-201 scintigraphy in patients unable to exercise. Circulation 1990; 82: 80-7.

69. Ogilby JD, Iskandrian AS, Untereker WJ, Heo J, Nguyen TN, Mercuro J. Effect of intravenous adenosine infusion on myocardial perfusion and function: hemodynamic/angiographic and scintigraphic study. Circulation 1992; 86: 887-95.

70. Martin TW, Seaworth JF, Johns JP, Pupa LE, Condos WR. Comparison of adenosine, dipyridamole and dobutamine in stress echocardiography. Ann Intern Med 1992; 116: 190-6.

71. Rossen JD, Quillen JE, Lopez AG, Stenberg RG, Jalman CL, Winniford MD
Comparison of coronary vasodilation with intravenous dipyridamole and adenosine. J Am Coll Cardiol 1991; 18: 485-91.

72. Mohiuddin SM, Gupta NC, Esterbrooks DJ, Hilleman DE. Safety profile of intravenous adenosine in cardiac imaging. Circulation 1990; 82(suppl III): III-731.

73. Nguyen T, Heo J, Paugh B, Iskandrian A. Atrioventricular block during adenosine-thallium imaging. Circulation 1990; 82(suppl III): III- 731.

74. Verani MS, Mahmarian JJ, Hixson JB, Boyce TM, Staudacher RA. Diagnosis of coronary artery disease by controlled coronary vasodilation with adenosine and thallium-201 scintigraphy in patients unable to exercise. Circulation 1990; 82: 80-7.

75. Nguyen T, Heo J, Vgilby JD, Iskandrian AS. Single photon emission computed tomography with thallium-201 during adenosine-induced coronary hyperemia; correlation with coronary arteriography, exercise thallium imaging and two-dimensional echocardiography. J Am Coll Cardiol 1990; 16: 1375-83.

76. Wackers FJT. Adenosine-thallium imaging: faster and better? J Am Coll Cardiol 1990; 16: 1384-6.

77. Gould KL, Lipscomb K, Hamilton GW. Physiologic basis for assessing critical coronary stenosis. Am J Cardiol 1974; 33: 87-94.

78. Khouri EM, Gregg DE, Lowensohn HS. Flow in the major branches of the left coronary artery during experimental coronary insufficiency in the unanesthetized dog. Circ Res 1968; 23: 99-109.

79. Goldstein RA, Kirkeeide RL, Demer LL, et al. Relation between geometric dimensions of coronary artery stenosis and myocardial perfusion reserve in man. $\mathrm{J}$ Clin Invest 1987; 79: 1473-8.

80. Klocke FJ. Measurements of coronary flow reserve: defining pathophysiology versus making decisions about patient care. Circulation 1987; 76: 1183-9. 